PROCEEDINGS OF THE AMERICAN MATHEMATICAL SOCIETY

Volume 124, Number 5, May 1996

\title{
IDENTIFYING CONGRUENCE SUBGROUPS OF THE MODULAR GROUP
}

\author{
TIM HSU
}

(Communicated by Ronald M. Solomon)

\begin{abstract}
We exhibit a simple test (Theorem 2.4) for determining if a given (classical) modular subgroup is a congruence subgroup, and give a detailed description of its implementation (Theorem 3.1). In an appendix, we also describe a more "invariant" and arithmetic congruence test.
\end{abstract}

\section{Notation}

We describe (conjugacy classes of) subgroups $\Gamma \subset \mathbf{P S L}_{2}(\mathbf{Z})$ in terms of permutation representations of $\mathbf{P S L}_{2}(\mathbf{Z})$, following Millington $[11,12]$ and Atkin and Swinnerton-Dyer [1].

We recall that a conjugacy class of subgroups of $\mathbf{P S L}_{2}(\mathbf{Z})$ is equivalent to a transitive permutation represention of $\mathbf{P S L}_{2}(\mathbf{Z})$. Such a representation can be defined by transitive permutations $E$ and $V$ which satisfy the relations

$$
1=E^{2}=V^{3} \text {. }
$$

The relations (1.1) are fulfilled by

$$
E=\left(\begin{array}{rr}
0 & 1 \\
-1 & 0
\end{array}\right), \quad V=\left(\begin{array}{rr}
1 & 1 \\
-1 & 0
\end{array}\right) .
$$

Alternately, such a representation can be defined by transitive permutations $L$ and $R$ which satisfy

$$
1=\left(L R^{-1} L\right)^{2}=\left(R^{-1} L\right)^{3},
$$

with the relations being fulfilled by

$$
L=\left(\begin{array}{ll}
1 & 1 \\
0 & 1
\end{array}\right), \quad R=\left(\begin{array}{ll}
1 & 0 \\
1 & 1
\end{array}\right) .
$$

One can also use permuations $E$ and $L$ such that

$$
1=E^{2}=\left(L^{-1} E\right)^{3},
$$

with $E$ and $L$ corresponding to the indicated matrices in (1.2) and (1.4), respectively.

Received by the editors September 1, 1994.

1991 Mathematics Subject Classification. Primary 20H05; Secondary 20 F05.

Key words and phrases. Congruence subgroups, classical modular group.

The author was supported by an NSF graduate fellowship and DOE GAANN grant \#P200A10022.A03.

(C)1996 American Mathematical Society 
The various notations can be translated using the following conversion table:

$$
\begin{array}{lll}
E=L R^{-1} L, & V=R^{-1} L, \\
L=E V^{-1}, & R=E V^{-2}, \\
& R=E^{-1} L^{-1} E . &
\end{array}
$$

Example 1.1. The permutations

$$
\begin{aligned}
& E=\left(\begin{array}{lll}
1 & 2
\end{array}\right)\left(\begin{array}{ll}
3 & 4
\end{array}\right)\left(\begin{array}{ll}
5 & 6
\end{array}\right)(7 \quad 8)(9 \text { 10), } \\
& V=\left(\begin{array}{llll}
1 & 3 & 5
\end{array}\right)\left(\begin{array}{lll}
2 & 7 & 4
\end{array}\right)\left(\begin{array}{l}
6 \\
8
\end{array}\right. \text { 9), }
\end{aligned}
$$

or, alternately,

$$
\begin{aligned}
& L=(14)(259108)(376) \text {, } \\
& R=\left(\begin{array}{llll}
1 & 7 & 9 & 10
\end{array}\right)(23)(458),
\end{aligned}
$$

describe a conjugacy class of subgroups of index 10 in $\mathbf{P S L}_{2}(\mathbf{Z})$.

Remark 1.2. Note that any concrete method of specifying a modular subgroup can easily be converted to permutation form. For instance, one way in which a modular subgroup $\Gamma$ might be specified is by a list of generators. Such a list can be converted into permutations as follows: First, use the Euclidean algorithm to express each generator matrix as a product of $L$ 's and $R$ 's, where $L$ and $R$ are the elements in (1.4). Then enumerate the cosets of $\Gamma$ in terms of these generators and presentation (1.3). This coset enumeration is easily converted into appropriate permutations $L$ and $R$. Similarly, any reasonable membership test for $\Gamma$ can be used to enumerate the cosets of $\Gamma$, with the same results as before.

\section{Congruence subgroups And the Level}

We recall the following definitions.

Definition 2.1. $\Gamma(N)$ is defined to be the group

$$
\left\{\gamma \in \mathbf{P S L}_{2}(\mathbf{Z}) \mid \gamma \equiv \pm I \quad(\bmod N)\right\} .
$$

$\Gamma(N)$ is the kernel of the natural projection from $\mathbf{P S L}_{2}(\mathbf{Z})$ to $\mathbf{S L}_{2}(\mathbf{Z} / N) /\{ \pm I\}$. We say that a modular subgroup $\Gamma$ is a congruence subgroup if $\Gamma$ contains $\Gamma(N)$ for some integer $N$. Otherwise, we say $\Gamma$ is a non-congruence subgroup.

An important invariant of (conjugacy classes of) modular subgroups is the following.

Definition 2.2. The level of a modular subgroup $\Gamma$, as specified by permutations $L$ and $R$, is defined to be the order of $L$ (or the order of $R$, since $L$ is conjugate to $\left.R^{-1}\right)$.

We need the following result, sometimes known as Wohlfahrt's Theorem (Wohlfahrt [13]).

Theorem 2.3. Let $N$ be the level of a modular subgroup $\Gamma$. $\Gamma$ is a congruence subgroup if and only if it contains $\Gamma(N)$.

Proof. This amounts to proving that, for congruence subgroups, our definition of the level is the same as the classical definition of the level. See Wohlfahrt [13]. 
Theorem 2.4. Let $\Gamma$ be a modular subgroup of level $N$, and let

$$
\left\langle L, R \mid r_{1}, r_{2}, \ldots\right\rangle
$$

be a presentation for $\mathbf{S L}_{2}(\mathbf{Z} / N) /\{ \pm I\}$ which is compatible with (1.4). Then $\Gamma$ is a congruence subgroup if and only if the representation of $\mathbf{P S L}_{2}(\mathbf{Z})$ induced by $\Gamma$ respects the relations $\left\{r_{i}\right\}$.

Proof. From Theorem 2.3, we only need to check if $\Gamma$ contains $\Gamma(N)$. Now, since $\Gamma(N)$ is normal in $\mathbf{P S L}_{2}(\mathbf{Z}), \Gamma$ contains $\Gamma(N)$ if and only if the normal kernel of $\Gamma$ contains $\Gamma(N)$. However, the normal kernel of $\Gamma$ is exactly the kernel of the representation induced by $\Gamma$, and since the relations $\left\{r_{i}\right\}$ generate $\Gamma(N)$ as their normal closure, the theorem follows.

Compare Magnus [9, Ch. III], Britto [4], Wohlfahrt [13], and Larcher [8]. Lang, Lim, and Tan [7] have also developed a congruence test; see the related paper Chan, Lang, Lim, and Tan [5].

Example 2.5. Suppose $\Gamma$ is the conjugacy class of subgroups specified by (1.10). Since $L$ has order 30 , we need to use a presentation for $\mathbf{S L}_{2}(\mathbf{Z} / 30) /\{ \pm I\}$. We find that $\mathbf{S L}_{2}(\mathbf{Z} / 30) /\{ \pm I\}$ has a presentation with defining relations

$$
\begin{gathered}
1=L^{30}, \\
1=\left[L^{2}, R^{15}\right]=\left[L^{3}, R^{10}\right]=\left[L^{5}, R^{6}\right]
\end{gathered}
$$

in addition to the relations in (1.3). (The commutator $[x, y]$ is defined to be $x^{-1} y^{-1} x y$, so $1=[x, y]$ means " $x$ commutes with $y$ ".) Only the commutator relations (2.4) need to be checked. However,

$$
L^{2}=\left(\begin{array}{lllll}
2 & 9 & 8 & 5 & 10
\end{array}\right)\left(\begin{array}{lll}
3 & 6 & 7
\end{array}\right),
$$

which does not commute with

$$
R^{15}=(23),
$$

so $\Gamma$ is a non-congruence subgroup. (It is worth mentioning that Larcher's results also imply that $\Gamma$ is non-congruence, since $L$ does not contain a 30 -cycle.)

Remark 2.6. The results in this section extend essentially verbatim to the Bianchi groups $\mathbf{S L}_{2}\left(O_{d}\right)$, where $O_{d}$ is the ring of algebraic integers of an imaginary quadratic field $\mathbf{Q}[\sqrt{-d}]$ with class number 1. (See Fine $[6]$ for more on the Bianchi groups.) However, for practical use, one needs a uniform presentation of $\mathbf{S L}_{2}\left(O_{d}\right) / \mathfrak{A}$ for $\mathfrak{A}$ any ideal of $O_{d}$.

\section{IMPLEMENTATION}

To assure the reader that the procedure described by Theorem 2.4 is practical, we provide the following detailed algorithm. Suppose we are given a subgroup $\Gamma$ of finite index in $\mathbf{P S L} \mathbf{L}_{2}(\mathbf{Z})$.

1. Describe $\Gamma$ in terms of permutations $L$ and $R$. If necessary, use conversion (1.7), conversion (1.8), or another similar conversion. (See also Remark 1.2.)

2. Let $N$ be the order of $L$, and let $N=e m$, where $e$ is a power of 2 and $m$ is odd.

3. We have three cases:

(a) $N$ is odd: $\Gamma$ is a congruence subgroup if and only if the relation

$$
1=\left(R^{2} L^{-\frac{1}{2}}\right)^{3}
$$


is satisfied, where $\frac{1}{2}$ is the multiplicative inverse of $2 \bmod N$.

(b) $N$ is a power of 2: Let $S=L^{20} R^{\frac{1}{5}} L^{-4} R^{-1}$, where $\frac{1}{5}$ is the multiplicative inverse of $5 \bmod N$. $\Gamma$ is a congruence subgroup if and only if the relations

$$
\begin{gathered}
\left(L R^{-1} L\right)^{-1} S\left(L R^{-1} L\right)=S^{-1}, \\
S^{-1} R S=R^{25} \\
1=\left(S R^{5} L R^{-1} L\right)^{3}
\end{gathered}
$$

are satisfied.

(c) Both e and $m$ are greater than 1:

(i) Let $\frac{1}{2}$ be the multiplicative inverse of $2 \bmod m$, and let $\frac{1}{5}$ be the multiplicative inverse of $5 \bmod e$.

(ii) Let $c$ be the unique integer $\bmod N$ such that $c \equiv 0(\bmod e)$ and $c \equiv 1$ $(\bmod m)$, and let $d$ be the unique integer $\bmod N$ such that $d \equiv 0(\bmod m)$ and $d \equiv 1(\bmod e)$.

(iii) Let $a=L^{c}, b=R^{c}, l=L^{d}, r=R^{d}$, and let $s=l^{20} r^{\frac{1}{5}} l^{-4} r^{-1}$.

(iv) $\Gamma$ is a congruence subgroup if and only if the relations

$$
\begin{gathered}
1=[a, r], \\
1=\left(a b^{-1} a\right)^{4}, \\
\left(a b^{-1} a\right)^{2}=\left(b^{-1} a\right)^{3}, \\
\left(a b^{-1} a\right)^{2}=\left(b^{2} a^{-\frac{1}{2}}\right)^{3}, \\
\left(l r^{-1} l\right)^{-1} s\left(l r^{-1} l\right)=s^{-1}, \\
s^{-1} r s=r^{25} \\
\left(l r^{-1} l\right)^{2}=\left(s r^{5} l r^{-1} l\right)^{3}
\end{gathered}
$$

are satisfied.

Theorem 3.1. The above procedure determines if $\Gamma$ is a congruence subgroup.

Before proving Theorem 3.1, we need an algebraic trick (Lemma 3.2) and some known results (Lemma 3.3, due to Behr and Mennicke [2]; and Lemma 3.4, due to Mennicke [10]).

Lemma 3.2 (Braid trick). Let $x$ and $y$ be elements which generate a group $G$ and satisfy the relation

$$
(x y x)^{2}=(y x)^{3} .
$$

Then the element $(x y x)^{2}=(y x)^{3}$ is central in G. Furthermore,

$$
x y x=y x y
$$

and

$$
(x y x)^{-1} x(x y x)=y .
$$

We call this the "braid trick" because (3.2) is the defining relation for the 3 -string braid group.

Proof. The elements $X=x y x$ and $Y=y x$ also generate $G$, and the element $Z=(x y x)^{2}=(y x)^{3}=X^{2}=Y^{3}$ commutes with both $X$ and $Y$, so $Z$ is central. (3.2) and (3.3) follow from cancellation in $x y x x y x=y x y x y x$. 
Lemma 3.3. Let $m$ be an odd integer, and let $\frac{1}{2}$ be the multiplicative inverse of 2 $\bmod m . \mathbf{S L}_{2}(\mathbf{Z} / m)$ is isomorphic to

$$
\begin{aligned}
G=\langle a, b| & \\
& 1=a^{m}, \\
& 1=\left(a b^{-1} a\right)^{4}, \\
& \left(a b^{-1} a\right)^{2}=\left(b^{-1} a\right)^{3}, \\
& \left.\left(a b^{-1} a\right)^{2}=\left(b^{2} a^{-\frac{1}{2}}\right)^{3}\right\rangle .
\end{aligned}
$$

Relations (3.4)-(3.7) are fulfilled by $a=\left(\begin{array}{ll}1 & 1 \\ 0 & 1\end{array}\right)$ and $b=\left(\begin{array}{ll}1 & 0 \\ 1 & 1\end{array}\right)$ in $\mathbf{S L}_{2}(\mathbf{Z} / m)$.

Proof. $G$ is equivalent to Behr and Mennicke's presentation [2, (2.12)] by the following Tietze transformations. Add generators $A=b$ and $B=a b^{-1} a$. Applying the braid trick to (3.6), we get that $B^{2}$ is central, and from (3.2), we also get that

$$
B A=b^{-1} a \text {. }
$$

(3.8) implies that $a=A B A$, which means that we can eliminate $a$ and $b$.

Using (3.3), (3.8), and the centrality of $B^{2}$, we see that (3.4)-(3.6) become

$$
\begin{aligned}
& 1=A^{m}=B^{4}, \\
& B^{2}=(A B)^{3},
\end{aligned}
$$

so it remains to convert (3.7) to Behr and Mennicke's form. However, applying (3.3), we have

$$
B^{2}=\left(b^{2} a^{-\frac{1}{2}}\right)^{3}=\left(A^{2} B^{-1} A^{\frac{1}{2}} B\right)^{3},
$$

so, using $1=B^{8}$ and the centrality of $B^{2}$,

$$
1=\left(A^{2} B^{-1} A^{\frac{1}{2}} B\right)^{3} B^{6}=\left(A^{2} B A^{\frac{1}{2}} B\right)^{3} .
$$

Lemma 3.4. Let $e=2^{n}$, let $\frac{1}{5}$ be the multiplicative inverse of $5 \bmod e$, and let $s=l^{20} r^{\frac{1}{5}} l^{-4} r^{-1} . \mathbf{S L}_{2}(\mathbf{Z} / e)$ is isomorphic to

$$
\begin{aligned}
G=\langle l, r| & \\
& 1=l^{e}, \\
& 1=\left(l r^{-1} l\right)^{4}, \\
& \left(l r^{-1} l\right)^{2}=\left(r^{-1} l\right)^{3}, \\
& \left(l r^{-1} l\right)^{-1} s\left(l r^{-1} l\right)=s^{-1}, \\
& s^{-1} r s=r^{25}, \\
& \left.\left(l r^{-1} l\right)^{2}=\left(s r^{5} l r^{-1} l\right)^{3}\right\rangle .
\end{aligned}
$$

Relations (3.13)-(3.18) are fulfilled by $l=\left(\begin{array}{ll}1 & 1 \\ 0 & 1\end{array}\right), r=\left(\begin{array}{ll}1 & 0 \\ 1 & 1\end{array}\right)$, and $s=\left(\begin{array}{ll}5 & 0 \\ 0 & \frac{1}{5}\end{array}\right)$ in $\mathbf{S L}_{2}(\mathbf{Z} / e)$. 
Proof. As the reader may verify, the relations (3.13)-(3.18) and $s=l^{20} r^{\frac{1}{5}} l^{-4} r^{-1}$ are satisfied in $\mathbf{S L}_{2}(\mathbf{Z} / e)$, so it suffices to show that $G$ is a homomorphic image of Mennicke's presentation [10, p. 210]. Add generators $A=r, B=l r^{-1} l$, and $T=s$. Applying the braid trick to (3.15), we get that $B^{2}$ is central, $B A=r^{-1} l$, and $l$ is conjugate to $A^{-1}$. As in the proof of the previous lemma, we can then eliminate generators $l$ and $r$. Then (3.13), (3.14), (3.15), (3.16), (3.17), and (3.18) become Mennicke's relations $(\mathrm{X}),(\mathrm{Y}),(\mathrm{P}),(\mathrm{Z}),(\mathrm{Q})$, and $(\mathrm{R})$, respectively.

For Lemma 3.5, we consider the following relations:

$$
\begin{gathered}
1=L^{N}, \\
1=[a, r], \\
1=[b, l], \\
1=\left(a b^{-1} a\right)^{4}, \\
\left(a b^{-1} a\right)^{2}=\left(b^{-1} a\right)^{3}, \\
\left(a b^{-1} a\right)^{2}=\left(b^{2} a^{-\frac{1}{2}}\right)^{3}, \\
1=\left(l r^{-1} l\right)^{4}, \\
\left(l r^{-1} l\right)^{2}=\left(r^{-1} l\right)^{3}, \\
\left(l r^{-1} l\right)^{-1} s\left(l r^{-1} l\right)=s^{-1}, \\
s^{-1} r s=r^{25}, \\
\left(l r^{-1} l\right)^{2}=\left(s r^{5} l r^{-1} l\right)^{3} .
\end{gathered}
$$

All notation is as described in (2) and (3c)(i-iii) of the algorithm. Note that $1=L^{N}$ implies that $L=a l$ and $R=b r$.

Lemma 3.5. $\mathbf{S L}_{2}(\mathbf{Z} / N)$ has a presentation with generators $L$ and $R$, and defining relations (3.19)-(3.29). The relations are fulfilled by $L=\left(\begin{array}{ll}1 & 1 \\ 0 & 1\end{array}\right)$ and $R=\left(\begin{array}{ll}1 & 0 \\ 1 & 1\end{array}\right)$ in $\mathbf{S L}_{2}(\mathbf{Z} / N)$.

Proof. The Chinese Remainder Theorem implies that

$$
\mathbf{S L}_{2}(\mathbf{Z} / N) \cong \mathbf{S L}_{2}(\mathbf{Z} / m) \times \mathbf{S L}_{2}(\mathbf{Z} / e) \text {. }
$$

It also follows from the Chinese Remainder Theorem that, if $L=\left(\begin{array}{ll}1 & 1 \\ 0 & 1\end{array}\right)$ and $R=$ $\left(\begin{array}{ll}1 & 0 \\ 1 & 1\end{array}\right)$ in $\mathbf{S L}_{2}(\mathbf{Z} / N)$, the $\mathbf{S L}_{2}(\mathbf{Z} / m)$ factor is precisely $\langle a, b\rangle$ and the $\mathbf{S L}_{2}(\mathbf{Z} / e)$ factor is precisely $\langle l, r\rangle$. Therefore, the above relations are satisfied in $\mathbf{S L}_{2}(\mathbf{Z} / N)$.

On the other hand, since (3.19) implies (3.4) and (3.13), comparison with Lemmas 3.3 and 3.4 shows that the above presentation is the direct product of $\mathbf{S L}_{2}(\mathbf{Z} / m)$ and $\mathbf{S L}_{2}(\mathbf{Z} / e)$. The lemma follows.

Proof of Theorem 3.1. After steps 1 and 2 of the procedure, we know that the relations

$$
\begin{gathered}
1=L^{N}, \\
1=\left(L R^{-1} L\right)^{2}, \\
1=\left(R^{-1} L\right)^{3}
\end{gathered}
$$


must be satisfied. From Theorem 2.4, we see that if (3.31)-(3.33) and (A) (resp. (B), (C)) are defining relations for $\mathbf{S L}_{2}(\mathbf{Z} / N) /\{ \pm I\}$ when $N$ is odd (resp. $N$ is a power of 2 , and $e$ and $m$ are greater than 1 ), then Theorem 3.1 follows. Comparing (A) and Lemma 3.3, with $a=L$ and $b=R$, and comparing (B) and Lemma 3.4, with $l=L$ and $r=R$, the first two cases follow easily, so it remains to check the third.

Comparing (C) and (3.19)-(3.29), we see that it is enough to show that given (3.31)-(3.33) and (3.19)-(3.29), the relations (3.21), (3.25), and (3.26) are redundant. First, (3.31), (3.32), (3.20), and (3.21) give us

$$
\begin{aligned}
1 & =\left(L R^{-1} L\right)^{4} \\
& =\left(a l r^{-1} b^{-1} a l\right)^{4} \\
& =\left(a b^{-1} a\right)^{4}\left(l r^{-1} l\right)^{4},
\end{aligned}
$$

which means that (3.22) implies (3.25). Similarly, (3.31), (3.32), (3.33), (3.20), and (3.21) imply

$$
\begin{aligned}
\left(L R^{-1} L\right)^{2} & =\left(R^{-1} L\right)^{3}, \\
\left(a b^{-1} a\right)^{2}\left(l r^{-1} l\right)^{2} & =\left(b^{-1} a\right)^{3}\left(r^{-1} l\right)^{3},
\end{aligned}
$$

which means that (3.23) implies (3.26). Finally, since (3.32), (3.33), and the braid trick (3.3) imply that $L$ is conjugate to $R^{-1}$, we can eliminate (3.21), since it is implied by (3.20).

For hand calculations, and for further study, we note the following relations which occur in $\mathbf{S L}_{2}(\mathbf{Z} / N)$ :

$$
\begin{aligned}
& \left(S L_{2}\right) \\
& Z=\left(L R^{-1} L\right)^{2}=\left(R^{-1} L\right)^{3}, 1=Z^{2}, \\
& \text { (level) } \\
& 1=L^{N}=R^{N}, \\
& (a b \equiv 0 \quad(\bmod N)) \\
& (a b \equiv-1 \quad(\bmod N)) \\
& 1=\left[L^{a}, R^{b}\right], \\
& (a b \equiv-2 \quad(\bmod N)) \\
& \left(L^{a} R^{b}\right)^{3}=Z, \\
& \left(L^{a} R^{b}\right)^{2}=Z .
\end{aligned}
$$

It has been verified by coset enumeration that the relations $\left(S L_{2}\right)$, (level), and $(a b \equiv 0(\bmod N))$ are defining relations when $N \mid 360$. This means that if the level $N$ divides 360 , the congruence test reduces to checking that the relations $(a b \equiv 0(\bmod N))$ are satisfied.

\section{ACKNOWLEDGEMENTS}

The author would like to thank J. H. Conway and the referee for many helpful comments and suggestions.

\section{Appendix A. An ARITHMETIC CONGRUENCE TEST}

In this appendix, we present an arithmetic and "invariant" congruence test which uses the Ihara modular group $\mathbf{S L}_{2}\left(\mathbf{Z}\left[\frac{1}{p}\right]\right)$. 
We begin by quoting the following result (Theorem A.1) of J. Mennicke [10]. (Note that Mennicke's Schur multiplier calculation and subsequent argument require the repairs described in F.R. Beyl [3, §5], but the main result still holds.) Let $N$ be an integer, let $p$ be a prime not dividing $N$, let $R_{N}$ be the kernel in $\mathbf{S L}_{2}\left(\mathbf{Z}\left[\frac{1}{p}\right]\right)$ resulting from reduction $\bmod N$, and let $Q_{N}$ be the normal closure of $L^{N}$ in $\mathbf{S L}_{2}\left(\mathbf{Z}\left[\frac{1}{p}\right]\right)$.

Theorem A.1. $R_{N}=Q_{N}$.

Let $\Gamma$ be a modular subgroup of level $N$ and index $m$ in $\mathbf{S L}_{2}(\mathbf{Z})$. Consider the commutative diagram in Figure A.1.

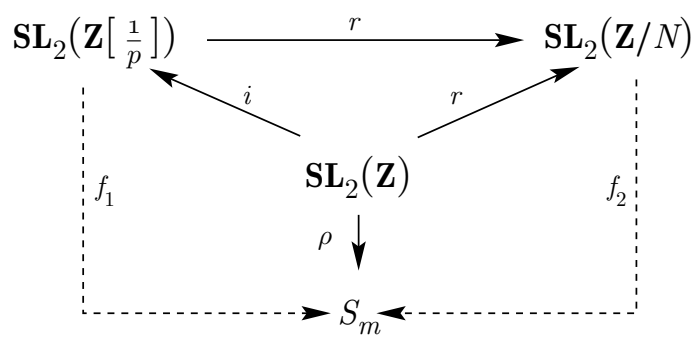

Figure A.1. Commutative diagram for Theorem A.2

Here, $S_{m}$ is the symmetric group on $m$ objects (the cosets of $\Gamma$ in $\mathbf{S L}_{2}(\mathbf{Z})$ ), $r$ is reduction mod $N, i$ is inclusion, and $\rho$ is the permutation representation of $\mathbf{S L}_{2}(\mathbf{Z})$ induced by $\Gamma$. Note that $f_{2}$ exists if and only if $\Gamma$ is a congruence subgroup, and that such an $f_{2}$ is uniquely determined.

The setup in Figure A.1 provides us with an invariant congruence test.

Theorem A.2. In the notation of Figure A.1, a map $f_{1}$ exists if and only if $f_{2}$ exists. In other words, $\Gamma$ is congruence if and only if $\rho$ can be factored through inclusion in $\mathbf{S L}_{2}\left(\mathbf{Z}\left[\frac{1}{p}\right]\right)$.

Proof. If $f_{2}$ exists, let $f_{1}=f_{2} r$. Conversely, if $f_{1}$ exists, since $L^{N}$ is in the kernel of $\rho, L^{N}$ must be in the kernel of $f_{1}$, so in fact, $f_{1}$ is well defined on

$$
\mathbf{S L}_{2}\left(\mathbf{Z}\left[\frac{1}{p}\right]\right) / Q_{N}=\mathbf{S L}_{2}\left(\mathbf{Z}\left[\frac{1}{p}\right]\right) / R_{N} \cong \mathbf{S L}_{2}(\mathbf{Z} / N)
$$

which means that $f_{1}$ defines an appropriate map $f_{2}$.

Corollary A.3. In Figure A.1, $f_{1}$ is determined uniquely if it exists.

One curious feature of Theorem A.2 is that if we know a given family of modular subgroups all have levels relatively prime to $p$, then we can handle all of them in a uniform manner. This is the principle behind Behr and Mennicke's presentation of $\mathbf{S L}_{2}(\mathbf{Z} / N)$ for $N$ odd, as these cases can be handled in $\mathbf{S L}_{2}\left(\mathbf{Z}\left[\frac{1}{2}\right]\right)$.

We also note that if we fix the level $N$, then we can choose any $p$ not dividing $N$ to use in Theorem A.2. This leads to the following idea: For a given family of modular subgroups of level $N$, it seems plausible that one might be able to reduce the extensibility of $\rho$ to the question of whether there exists a $p$ which satisfies certain congruences mod $N$. Dirichlet's theorem might then be used to find a $p$ which satisfies those congruences. 


\section{REFERENCES}

[1] A. O. L. Atkin and H. P. F. Swinnerton-Dyer, Modular forms on noncongruence subgroups, Proc. Symp. Pure Math., Combinatorics (Providence) (T. S. Motzkin, ed.), vol. 19, AMS, Providence, 1971, pp. 1-26. MR 49:2550

[2] H. Behr and J. Mennicke, A presentation of the groups PSL(2,p), Can. J. Math. 20 (1968), 1432-1438. MR 38:4566

[3] F. R. Beyl, The Schur multiplicator of $S L(2, Z / m Z)$ and the congruence subgroup property, Math. Z. 191 (1986), 23-42. MR 87b:20071

[4] J. Britto, On the construction of non-congruence subgroups, Acta Arith. XXXIII (1977), 261-267. MR 56:12142

[5] S.-P. Chan, M.-L. Lang, C.-H. Lim, and S.-P. Tan, Special polygons for subgroups of the modular group and applications, Internat. J. Math. 4 (1993), no. 1, 11-34. MR 94j:11045

[6] B. Fine, Algebraic theory of the Bianchi groups, Marcel Dekker, Inc., New York, 1989. MR 90h:20002

[7] M.-L. Lang, C.-H. Lim, and S.-P. Tan, An algorithm for determining if a subgroup of the modular group is congruence, preprint, 1992.

[8] H. Larcher, The cusp amplitudes of the congruence subgroups of the classical modular group, Ill. J. Math. 26 (1982), no. 1, 164-172. MR 83a:10040

[9] W. Magnus, Noneuclidean tesselations and their groups, Academic Press, 1974. MR 50:4774

[10] J. Mennicke, On Ihara's modular group, Invent. Math. 4 (1967), 202-228. MR 37:1485

[11] M. H. Millington, On cycloidal subgroups of the modular group, Proc. Lon. Math. Soc. 19 (1969), 164-176. MR 40:1484

[12] _ Subgroups of the classical modular group, J. Lon. Math. Soc. 1 (1969), 351-357. MR 39:5477

[13] K. Wohlfahrt, An extension of F. Klein's level concept, Ill. J. Math. 8 (1964), 529-535. MR 29:4805

Department of Mathematics, Princeton University, Princeton, New Jersey 08544

E-mail address: timhsu@math.princeton.edu

Current address: Department of Mathematics, University of Michigan, Ann Arbor, Michigan 48109

E-mail address: timhsu@math.1sa.umich.edu 